\title{
Using ESSENCE-FL for Situational Awareness after National Reports of Increased Enterovirus D68 (EV-D68) Infections with Severe Outcomes, September 2014
}

\author{
David Atrubin* \\ Florida Department of Health, Tampa, FL, USA
}

\section{Objective}

To provide situational awareness using Florida's syndromic surveillance system during a 2014 outbreak of EV-D68 in other regions of the country

\section{Introduction}

The Electronic Surveillance System for the Early Notification of Community-Based Epidemics (ESSENCE-FL) receives daily (or bi-hourly) data from 184 emergency departments (ED) from around Florida. Additionally, 30 urgent care centers submit daily data to the system. These 214 facilities are grouped together in an acute care data source category. Five to six days after the start of each school year in Florida, ESSENCE-FL shows increased respiratory illness visits in the school aged population. Previous analyses of these data have shown that this increase is a result of increased transmission of the common cold among school children. In early September 2014, during this sustained yearly increase in respiratory visits, reports of more severe infection caused by Enterovirus D68 (EV-D68) in children in other parts of the country began circulating. Public health officials in Florida, as well as the media, questioned whether children in the state were being infected by this virus capable of causing more severe illness, especially among asthmatics. As is the case with many incipient outbreaks, syndromic surveillance played an integral role in early efforts to detect the presence of this illness. The task of providing situational awareness during this period was complicated by this outbreak coinciding with the start of the school year.

\section{Methods}

On September 8, 2014, ESSENCE-FL staff were made aware of clusters of EV-D68 occurring in Kansas City, Missouri and Chicago. An early release edition of the Centers for Disease Control and Prevention's Morbidity and Mortality Weekly Report (September 8, 2014) was reviewed and provided critical information about disease presentation and the characteristics of the population infected with EV-D68. ${ }^{1}$ Using the 184 hospitals only, a time series graph was created to look for statistical aberrations in the percentage of 0-16 year olds being admitted to the hospital. The discharge disposition category field provides an indication of the severity of the illness in the ED patient. ESSENCE-FL subsyndromes were analyzed to look for visits in excess of normal levels for the categories of DifficultyBreathing, Wheezing and Asthma. Specific time series graphs were created in an effort to detect an increase in 0-16 year old asthmatics being admitted to hospitals.

\section{Results}

As of September 9, 2014, no increase in admit percentage was observed in children 0-16 years of age. Likewise, no levels of Respiratory syndrome or DifficultyBreathing, Wheezing, or Asthma subsyndromes were seen in excess of typical levels for this time of year for this age group. Additionally, The Florida Department of Health received no reports of increased hospital admissions related to this seasonal increase in respiratory visits typically seen at this time of year.

\section{Conclusions}

No evidence of increased illness due to EV-D68 was observed in Florida's syndromic surveillance ED data at the time of this writing. Syndromic surveillance systems often prove their worth as much by what is not found, rather than what is found. While these systems do not replace open communication with hospitals and other providers, there is a necessity to have near real-time surveillance when outbreaks are occurring - even elsewhere in the country or world. Understanding the periodicity in your data also facilitates the ability to provide accurate situational awareness. Having a specific data field (the discharge disposition) that allowed epidemiologists to gauge the level of severity of illness in an ED patient proved to be very beneficial in this circumstance.

\section{Keywords}

Enterovirus; Respiratory illness; school year; Emergency department data; admit data

\section{Acknowledgments}

Johns Hopkins University Applied Physics Laboratory

\section{References}

1. CDC. Severe Respiratory Illness Associated with Enterovirus D68 Missouri and Illinois, 2014. MMWR Sept 8, 2014;63:Early Release.

2. CDC Clusters of Acute Respiratory Illness Associated with Human Enterovirus 68 --Asia, Europe, and United States, 2008-2010. MMWR 2011;60:1301-4.

\author{
*David Atrubin \\ E-mail: david.atrubin@flhealth.gov
}

